\title{
Camerawork for Comics Generated from Visitors' Experiences in a Virtual Museum
}

\author{
Ruck Thawonmas and Kohei Kato \\ Intelligent Computer Entertainment Laboratory, \\ Graduate School of Science and Engineering, Ritsumeikan University. \\ 1-1-1 Nojihigashi, Kusatsu, Shiga, 525-8577, Japan \\ ruck@ci.ritsumei.ac.jp
}

\begin{abstract}
We present a camerawork module for representing events in a virtual museum with a comic style. The module uses four basic camera shots, i.e., internal, external, apex, and group, to compose a shot sequence of two main events happening in a museum, i.e., move and stay. This module is fully implemented in a comic generation system we have been developing for summarizing user experiences in Second Life. Visual comparison, with a baseline camerawork, confirms the effectiveness of the proposed camerawork module.
\end{abstract}

Keywords: camerawork, comic, virtual museum, metaverse, Second Life.

\section{Introduction}

A comic style is a promising representation for summarizing various kinds of user experiences in an entertaining fashion. Applications of the comic-style representation include summarization of activities in a conference [1], daily activities [2], video sequences [3, 4], and game-play activities [5-8]. Comic-style summaries facilitate perceiving of main events, augmenting of personal memories, and promoting of communication among user communities. In this paper, we describe a part of our current project on summarizing user experiences in a museum located in metaverse such as Second Life (SL) [9, 10]. In particular, we focus on how to perform camerawork in such an environment. In addition, we propose four basic camera shots and three camera idioms, each of which specifies a sequence of those basic camera shots for a certain situation.

Previously, we proposed in [11] and [12] a camerawork editor and an automatic camerawork module, respectively. The former allows the viewer to manually edit the camerawork of a generated comic while the latter automatically decides the camerawork of each frame based on rules derived from an analysis of online-game web-comics. However, the camerawork of online-game experiences, where major activities are related to fights against monsters and acquisitions of items, is different from that of user experiences in virtual museums, where major activities are simply moving around and staying to view exhibits therein.

In this paper, as our first step, we follow He et al. [13] in deriving basic shots and idioms beforehand. We then use them for deciding the camerawork of a selected comic frame and perform user evaluation to see their effectiveness. Story control as done in [14] and optimization of camera parameters is, however, beyond the scope of this paper. 


\section{Camerawork Module}

The proposed camerawork module decides a shot sequence for each scene according to one of the three idioms: move idiom, single-view idiom, and multiple-view idiom. For move scenes where the avatar continuously moves, the move idiom is used. For stay scenes where the avatar stays in order to view a nearby exhibit, the camerawork module uses the single-view idiom when the number of exhibits near the avatar is one and the multiple-view idiom when there are two or more nearby exhibits. Four basic camera shots inspired by the work of He et al. [13], i.e., internal, external, apex, and group, are used in these idioms. While basic shots in [13] were decided for avatars and interactions among them, our basic shots focus on the user avatar and its interactions between nearby exhibits or objects.

Figure 1 shows the camera placement and an example shot for internal, external, apex, and group, respectively, where $a$ and $o$ denote the avatar and an object of interest, respectively. The camera direction of each of these shots is towards the avatar's head, the second argument' center point, the center point between the avatar and the object, and the center point between the two objects, respectively. For each camera shot, three camera parameters, i.e., the camera angle, camera position, and zoom position, are decided in advance. As mentioned in Sec. 1, optimization of these parameters is beyond the scope of this paper.

Figure 2 shows the shot sequence and an example of each of the three camera idioms. We decided their shot sequences based on two cinematographer heuristics discussed in [13]: avoid jump cuts and use establishing shots. The former heuristic establishes a distinction between any two adjacent scenes. The latter heuristic allows the viewer to see all involving objects in the initial shot of a scene. The description of each idiom is given as follows:

Move idiom establishes a scene with external $(a, o)$ so that object $o$ is seen over the shoulder of avatar $a$, where $o$ is a virtual point located at a certain distance ahead of the avatar on the avatar's direction line. Then $\operatorname{apex}(a, o)$ is used, in which the camera focus is directed towards the point between the avatar and the virtual point, in order to express the avatar's movement.

Single-view idiom establishes a scene with external $(a, o)$ and then switches to external $(o, a)$ in order to prevent shooting similar consecutive shots, where $o$ is the nearest exhibit within a given distance from the avatar or the aforementioned virtual point if there are no exhibits nearby.

Multiple-view idiom establishes a scene with $\operatorname{group}\left(a, o_{1}, o_{2}\right)$ in which the nearest exhibit $o_{1}$ and the second nearest exhibit $o_{2}$ are both seen over the shoulder of the avatar. Then, in order to make the scene more exciting and lively, this idiom uses a combination of internal shots as well as single-view idioms.

For a scene of interest, the camerawork of each of its frames is decided according to the basic shot, one by one from top to bottom, in the corresponding idiom until the last frame of this scene. If there are still remaining frames when the bottom basic shot has been reached, the camerawork of the first remaining frame will be decided according to the top basic shot again. This process is repeated until the camerawork of all frames is decided. 


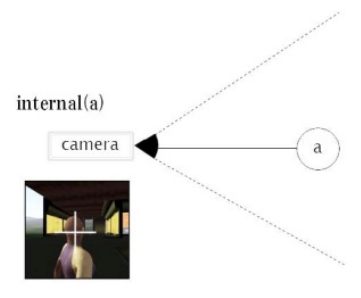

(a) Internal

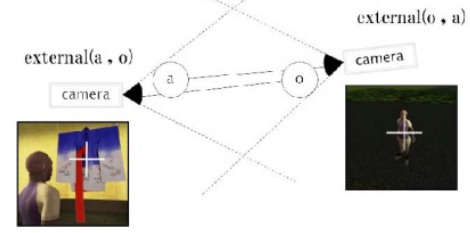

(b) External

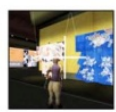

$\operatorname{group}(\mathrm{a}, 01,02)$

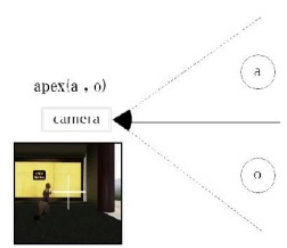

(c) Apex

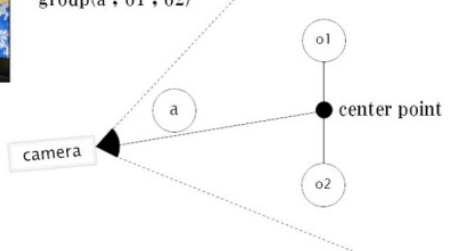

(d) Group

Fig. 1. Camera placement for the four basic camera-shots proposed in this work

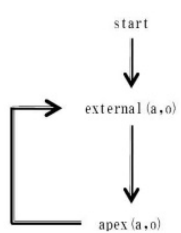

(a) Move idiom

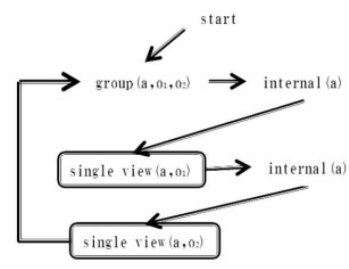

(c) Multiple-view idiom

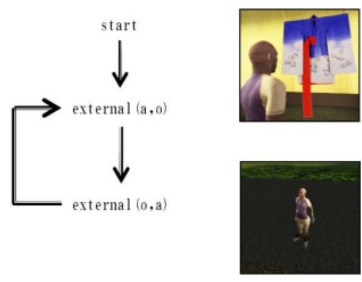

(b) Single-view idiom

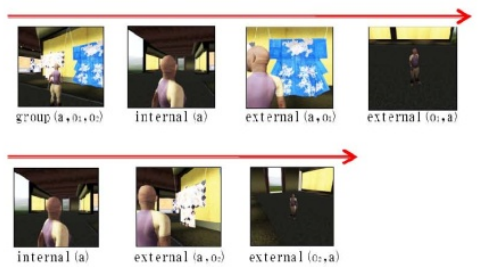

(d) Multiple-view shot sequence

Fig. 2. Shot sequence and example shots of the three camera idioms proposed in this work 


\section{Results and Discussions}

We fully implemented the proposed camerawork module in our comic generation system $[9,10]$ developed based on the open-source SL viewer program. For this work, we adopted a typical comic layout where the order to read is in the raster order, from top-left to bottom-right, and all frames have the same size.
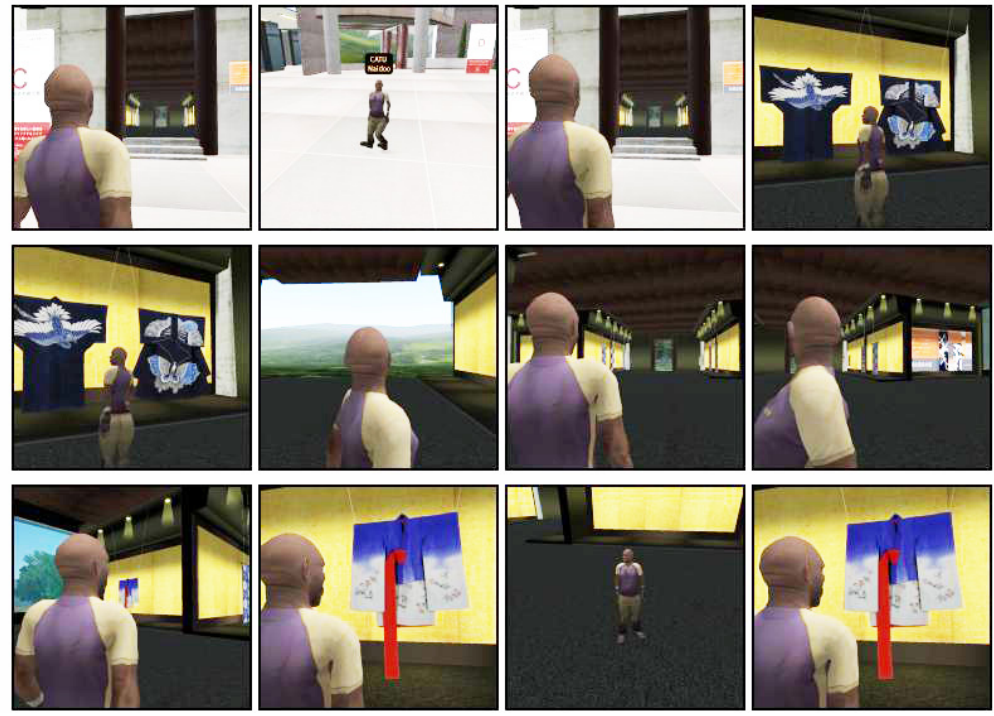

Fig. 3. Comic with the proposed camerawork
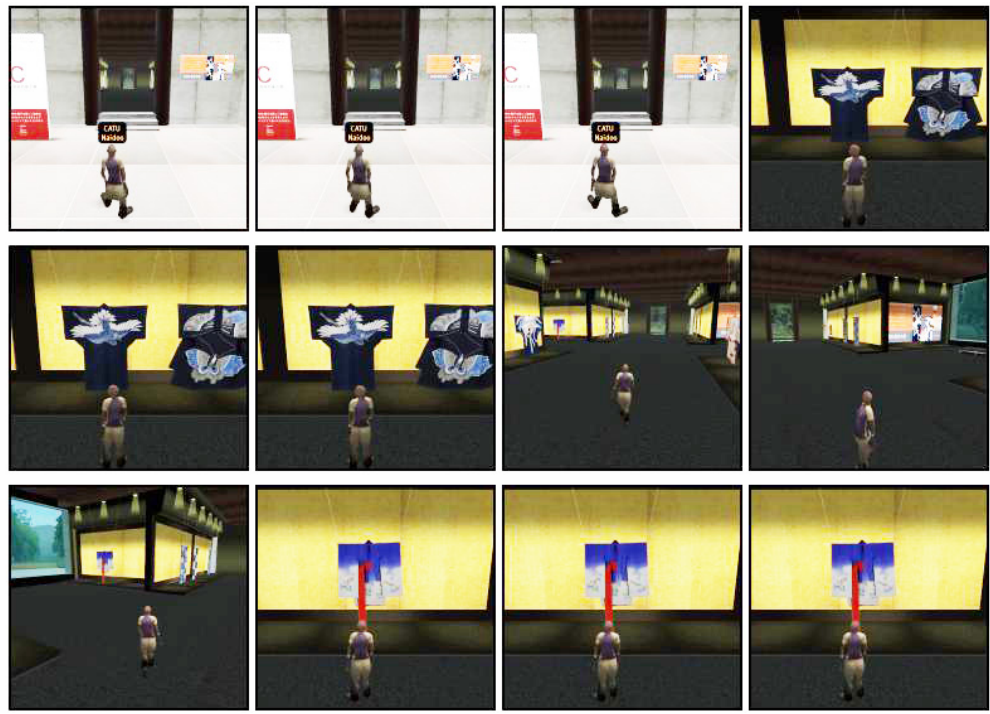

Fig. 4. Comic with the baseline camerawork 
In this work, we targeted user experiences at a SL museum ${ }^{1}$ designed and operated by members of the Global COE (Center of Excellence) Program Digital Humanities Center for Japanese Arts and Culture of Ritsumeikan University. This museum exhibits Kaga Okunizome Dyeing, kimono and bedding from the Ishikawa region in Japan during the latter part of the Edo period until the beginning of the Showa period. However, we note that our system is also applicable to other virtual museums, galleries, and exhibitions in SL as well as other metaverse.

Figures 3 and 4 visually compared comics whose camerawork was decided by the proposed camerawork module and by a baseline camerawork module, respectively. The baseline module used the same camera parameters as those of the SL viewer program. From these figures, one can see that similar consecutive frames exist in the comic with the baseline camerawork while this is not seen in the comic with the proposed camerawork.

\section{Conclusions and Future Work}

This paper described our camerawork module that decides the camerawork of each comic frame representing user experiences in a virtual museum. First, we decided the four basic camera shots, internal, external, apex, and group, such that the avatar movement and the avatar interactions between nearby exhibits are articulated in comic frames. Then we derived the three camera idioms, move, single view, multiple view, based on two well-known cinematographer heuristics avoid jump cuts and use establishing shots. Visual comparison confirmed the effectiveness of the proposed camerawork module in that the resulting comic was more exciting, lively and entertaining than that with the baseline camerawork.

Acknowledgement. This work is supported in part by the - MEXT Global COE Program - Digital Humanities Center for Japanese Arts and Cultures, Ritsumeikan University, and by Grant-in-Aid for Scientific Research (C), No. 23500192, the Japan Society for Promotion of Science.

\section{References}

1. Sumi, Y., Sakamoto, R., Nakao, K., Mase, K.: ComicDiary: Representing Individual Experiences in a Comics Style. In: Borriello, G., Holmquist, L.E. (eds.) UbiComp 2002. LNCS, vol. 2498, pp. 16-32. Springer, Heidelberg (2002)

2. Cho, S.B., Kim, K.J., Hwang, K.S.: Generating Cartoon-Style Summary of Daily Life with Multimedia Mobile Devices. In: Okuno, H.G., Ali, M. (eds.) IEA/AIE 2007. LNCS (LNAI), vol. 4570, pp. 135-144. Springer, Heidelberg (2007)

3. Calic, J., Gibson, D.P., Campbell, N.W.: Efficient Layout of Comic-like Video Summaries. IEEE Transactions on Circuits and Systems for Video Technology 17(7), 931-936 (2007)

${ }^{1}$ http://slurl.com/secondlife/rits¥\%20gcoe¥\%20jdh/167/189/22 
4. Tobita, H.: DigestManga: interactive movie summarizing through comic visualization. In: CHI. Extended Abstracts 2010, pp. 3751-3756 (2010)

5. Shamir, A., Rubinstein, M., Levinboim, T.: Generating Comics from 3D Interactive Computer Graphics. IEEE Computer Graphics and Applications 26(3), 53-61 (2006)

6. Shuda, T., Thawonmas, R.: Automatic Comic Generation Using Online-Game Play Log. Journal of Game Amusement Society 3(1), 40-45 (2009) ()in Japanese)

7. Chan, C.-J., Thawonmas, R., Chen, K.-T.: Automatic Storytelling in Comics: A Case Study onWorld ofWarcraft. In: CHI Extended Abstracts 2009, pp. 3589-3594 (2009)

8. Thawonmas, R., Tani, Y.: Frame Selection Using Iterative Grammatical Evolution for Automatic Comic Generation from Game Logs. In: Proc. of the 2011 IEEE Conference on Computational Intelligence and Games (CIG 2011), Seoul South Korea, August 31September 3 (2011)

9. Thawonmas, R., Fukumoto, A.: Frame Extraction Based on Displacement Amount for Automatic Comic Generation from Metaverse Museum Visit Log. In: Proc. of Int. Conf. on Intelligent and Interactive Multimedia - Systems and Services (IIMSS-2011), Piraeus, Greece, July 20-22 (2011)

10. Thawonmas, R., Shuda, T.: Frame Selection for Automatic Comic Generation from Museum Playlog in Metaverse. In: Proc. of IADIS International Conference IADIS Game and Entertainment Technologies 2011, Rome, Italy, July 22-24 (2011)

11. Thawonmas, R., Oda, K., Shuda, T.: Camerawork Editor for Automatic Comic Generation from Game Log. In: CD-ROM Proc. of Nicograph International 2009, Kanazawa, Japan, June 19-20 (2009)

12. Thawonmas, R., Oda, K., Shuda, T.: Rule-Based Camerawork Controller for Automatic Comic Generation from Game Log. In: Yang, H.S., Malaka, R., Hoshino, J., Han, J.H. (eds.) ICEC 2010. LNCS, vol. 6243, pp. 326-333. Springer, Heidelberg (2010)

13. He, L., Cohen, M.F., Salesin, D.H.: The Virtual Cinematographer: A Paradigm for Automatic Real-Time Camera Control and Directing. Computer Graphics 30, 217-224 (1996)

14. Jhala, A., Young, R.M.: Cinematic Visual Discourse: Representation, Generation, and Evaluation. IEEE Trans. Comput. Intellig. and AI in Games 2(2), 69-81 (2010) 